\title{
ON SUBTRACTIVE EXTENSION OF SUBSEMIMODULES OF SEMIMODULES
}

\author{
Jayprakash Ninu Chaudhari * and Dipak Ravindra Bonde **
}

\begin{abstract}
Let $R$ be a commutative semiring with $1_{R} \neq 0_{R}$. Characterization of subsemimodules, prime subsemimodules and primary subsemimodules which are subtractive extensions of $Q$-subsemimodules in $R$-semimodules are investigated.
\end{abstract}

\section{Introduction}

Let $R$ be a commutative semiring with $1_{R} \neq 0_{R}$. A commutative monoid $(M,+)$ with a scalar multiplication $R \times M \rightarrow M$, defined by $(r, x) \mapsto r x$ is called left $R$-semimodule if it satisfies the following conditions for all $r, r^{\prime} \in R$ and $x, y \in M$ :

1) $\left(r r^{\prime}\right) x=r\left(r^{\prime} x\right)$

2) $r(x+y)=r x+r y$

3) $\left(r+r^{\prime}\right) x=r x+r^{\prime} x$;

4) $1_{R} x=x$

5) $r 0_{M}=0_{M}=0_{R} x$.

Clearly every ring is a semiring and hence every left module over a ring $R$ is a left semimodule over a semiring $R$. Throughout by an $R$ semimodule we mean a left $R$-semimodule. Denote the sets of all nonnegative, and positive integers respectively by $\mathbb{Z}_{0}^{+}$, and $\mathbb{N}$. The set $\mathbb{Z}_{0}^{+}$ is a semiring under usual addition and multiplication of non-negative integers but it is not a ring. If $(M,+)$ is an idempotent commutative monoid, then $M$ is $\left(\mathbb{Z}_{0}^{+},+, \cdot\right)$-semimodule with scalar multiplication defined by $r m=0$ if $r=0$ and $r m=m$ if $r>0$ for all $r \in \mathbb{Z}_{0}^{+}$and $m \in$ $M$ [6, P.151]. A non-empty subset $N$ of an $R$-semimodule $M$ is called

Received June 14, 2012; Accepted January 11, 2013.

2010 Mathematics Subject Classification: Primary 16Y60.

Key words and phrases: semiring, subtractive subsemimodule, $Q$-subsemimodule, quotient semimodule, subtractive extension of a subsemimodule.

Correspondence should be addressed to Jayprakash Ninu Chaudhari, jnchaudhari@ rediffmail.com. 
subsemimodule of $M$ if $N$ is closed under addition and closed under scalar multiplication. A subsemimodule $N$ of an $R$-semimodule $M$ is called subtractive subsemimodule ( $=k$-subsemimodule) if $x, x+y \in N$, $y \in M$, then $y \in N$. A proper subsemimodule $N$ of an $R$-semimodule $M$ is said to be prime (primary) if $r m \in N, r \in R, m \in M$, then either $r M \subseteq N$ or $m \in N\left(r^{n} M \subseteq N\right.$ for some $n \in \mathbb{N}$ or $\left.m \in N\right)$. A proper subsemimodule $N$ of an $R$-semimodule $M$ is said to be weakly prime (weakly primary) if $0 \neq r m \in N, r \in R, m \in M$, then either $r M \subseteq N$ or $m \in N\left(r^{n} M \subseteq N\right.$ for some $n \in \mathbb{N}$ or $\left.m \in N\right)$. Consider $M=\left(\mathbb{Z}_{0}^{+},+\right)$ an $R$-semimodule where $R=\left(\mathbb{Z}_{0}^{+},+, \cdot\right)$. For $m \in M$, we denote the subsemimodule $\{a m \in M: a \in R\}$ of $M$ by $m \mathbb{Z}_{0}^{+}$.

Example 1.1. Consider a semiring $R=\left(\mathbb{Z}_{0}^{+},+, \cdot\right)$. Then

1) $9 \mathbb{Z}_{0}^{+}$is a primary subsemimodule of an $R$-semimodule $\left(\mathbb{Z}_{0}^{+},+\right)$but it is not a prime subsemimodule.

2) $4 \mathbb{Z}_{0}^{+}$is a weakly primary subsemimodule of an $R$-semimodule $\left(\mathbb{Z}_{0}^{+},+\right)$ but it is not a weakly prime subsemimodule.

3) $6 \mathbb{Z}_{0}^{+}$is neither a prime, primary, weakly prime nor a weakly primary subsemimodule of an $R$-semimodule $\left(\mathbb{Z}_{0}^{+},+\right)$.

4) $\{0\}$ is a weakly prime (weakly primary) subsemimodule of an $R$ semimodule $\left(\mathbb{Z}_{6},+_{6}\right)$ but it is not a prime (primary)subsemimodule.

A subsemimodule $N$ of an $R$-semimodule $M$ is called a $Q$-subsemimodule (= partitioning subsemimodule) if there exists a subset $Q$ of $M$ such that

1) $M=\cup\{q+N: q \in Q\}$.

2) if $q_{1}, q_{2} \in Q$, then $\left(q_{1}+N\right) \cap\left(q_{2}+N\right) \neq \emptyset \Leftrightarrow q_{1}=q_{2}$.

Let $N$ be a $Q$-subsemimodule of an $R$-semimodule $M$. Then $M / N_{(Q)}=$ $\{q+N: q \in Q\}$ forms an $R$-semimodule under the following addition

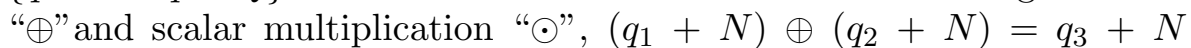
where $q_{3} \in Q$ is unique such that $q_{1}+q_{2}+N \subseteq q_{3}+N$, and $r \odot$ $\left(q_{1}+N\right)=q_{4}+N$ where $q_{4} \in Q$ is unique such that $r q_{1}+N \subseteq q_{4}$ $+N$. This $R$-semimodule $M / N_{(Q)}$ is called the quotient semimodule of $M$ by $N$ and denoted by $\left(M / N_{(Q)}, \oplus, \odot\right)$ or just $M / N_{(Q)}$. If $N$ is $Q$-subsemimodule of an $R$-semimodule $M$, then there exists a unique $q_{0} \in Q$ such that $q_{0}+N=N\left[3\right.$, Lemma 2.3]. This $q_{0}+N$ is the zero element of $M / N_{(Q)}$.

Chaudhari and Bonde [3], have introduced $Q$-subsemimodule and obtained a relation between subtractive subsemimodules and $Q$-subsemimodules. Some works on $Q$-subsemimodules, quotient semimodules, prime subsemimodules and maximal $k$-subsemimodules may be found in 
[2, 8 and 9]. Theory of $Q$-subsemimodules, prime subsemimodules, primary subsemimodules, direct sum of subsemimodules is recently studied by Chaudhari and Bonde [4 and 5].

In this paper, we introduce the notion of subtractive extension of a subsemimodule of an $R$-semimodule $M$ and prove its existence. Some characterizations of subtractive extension of $Q$-subsemimodule in $R$ semimodules are also obtained.

The following lemma will be used to prove our results.

Lemma 1.2. [3, Lemma1.3] Let $N$ be subsemimodule of an $R$-semimodule $M$ and $x, y \in M$ such that $x+N \subseteq y+N$. Then $x+z+N \subseteq$ $y+z+N$ and $r x+N \subseteq r y+N$ for all $z \in M, r \in R$.

\section{Subtractive extension of a subsemimodule}

In this section, we introduce the notion of subtractive extension of a subsemimodule of an $R$-semimodule $M$ and prove its existence.

Definition 2.1. Let $N$ be a subsemimodule of an $R$-semimodule $M$. A subsemimodule $A$ of $M$ with $N \subseteq A$ is said to be subtractive extension of $N$ if $x \in N, x+y \in A, y \in M$, then $y \in A$.

Every subtractive subsemimodule of an $R$-semimodule $M$ containing a subsemimodule $N$ is a subtractive extension of $N$. But the converse is not true.

ExAmple 2.2. Let $R=\left(\mathbb{Z}_{0}^{+},+, \cdot\right)$ be a semiring.

1) Consider $M=\left(\mathbb{Z}_{0}^{+} \cup\{\infty\}, \max \right)$ an $R$-semimodule. Let $N=$ $\{0,1,2,3,4,5\}$ be a subsemimodule of $M$. Now $A=\{0,1,2,3,4,5$, $6,7,8, \infty\}$ is a subsemimodule of $M$ with $N \subseteq A$. Clearly, if $x \in N, x+y \in A$ and $y \in M$, then $y \in A$. Hence $A$ is a subtractive extension of $N$. But $A$ is not a subtractive subsemimodule of $M$ because $\infty \in A, \infty+9 \in A$ and $9 \notin A$.

2) Consider $M=\left(\mathbb{Z}_{0}^{+},+\right)$an $R$-semimodule. For $m \in M$, we have $m \mathbb{Z}_{0}^{+}$is a $Q$-subsemimodule of $M$ where $Q=\{0,1,2,3, \ldots,(m-1)\}$. By [2, Theorem 3.2], $m \mathbb{Z}_{0}^{+}$is a subtractive subsemimodule of $M$. Hence $m \mathbb{Z}_{0}^{+}$is a subtractive extension of every subsemimodule $N$ of $M$ where $N \subseteq m \mathbb{Z}_{0}^{+}$.

Theorem 2.3. Let $N$ be a $Q$-subsemimodule of an $R$-semimodule $M$ and $A$ be a subsemimodule of $M$ with $N \subseteq A$. Denote $\widetilde{A}=\{x \in M$ : there exists $q+N \in M / N_{(Q)}$ such that $x \in q+N$ and $(q+$ 
$N) \cap A \neq \emptyset\}$. Then $\widetilde{A}$ is the smallest subtractive extension of $N$ containing $A$.

Proof. We have $q_{0}+N=N$ is the zero element of $M / N_{(Q)}$ and $N \subseteq A \Rightarrow q_{0} \in\left(q_{0}+N\right) \cap A \Rightarrow q_{0} \in \widetilde{A} \Rightarrow \widetilde{A} \neq \emptyset$. Let $x, y \in \widetilde{A}, r \in R$. Then there exist unique $q_{1}, q_{2} \in Q$ such that $x \in q_{1}+N, y \in q_{2}+N$ and $\left(q_{1}+N\right) \cap A \neq \emptyset,\left(q_{2}+N\right) \cap A \neq \emptyset$. Now $x+y \in\left(q_{1}+N\right) \oplus\left(q_{2}+N\right)=q+N$ where $q$ is a unique element of $Q$ such that $q_{1}+q_{2}+N \subseteq q+N$. Since $\left(q_{1}+N\right) \cap A \neq \emptyset$ and $\left(q_{2}+N\right) \cap A \neq \emptyset,(q+N) \cap A \neq \emptyset$. So $x+y \in \widetilde{A}$. Similarly $r x \in \widetilde{A}$. If $a \in A$, then there exists a unique $q \in Q$ such that $a \in q+N$. So $(q+N) \cap A \neq \emptyset$. Now $a \in \widetilde{A}$. Thus $\widetilde{A}$ is a subsemimodule of $M$ containing $A$. Let $x \in N, x+y \in \widetilde{A}, y \in M$. So $x+y \in q^{\prime}+N$ where $q^{\prime}+N \in M / N_{(Q)}$ and $\left(q^{\prime}+N\right) \cap A \neq \emptyset$. Since $N$ is a $Q$-subsemimodule of $M$, there exists a unique $q^{\prime \prime} \in Q$ such that $y \in q^{\prime \prime}+N$. As $x \in N \Rightarrow x+y \in q^{\prime \prime}+N$. So $\left(q^{\prime}+N\right) \cap\left(q^{\prime \prime}+N\right) \neq$ $\emptyset \Rightarrow q^{\prime}=q^{\prime \prime} \Rightarrow q^{\prime \prime}+N=q^{\prime}+N \Rightarrow\left(q^{\prime \prime}+N\right) \cap A=\left(q^{\prime}+N\right) \cap A \neq \emptyset$. Hence $y \in \widetilde{A}$. Thus $\widetilde{A}$ is a subtractive extension of $N$. Now let $B$ be any subtractive extension of $N$ containing $A$. Now $a \in \widetilde{A} \Rightarrow a \in q^{\prime \prime \prime}+N$ and $\left(q^{\prime \prime \prime}+N\right) \cap A \neq \emptyset \Rightarrow\left(q^{\prime \prime \prime}+N\right) \cap B \neq \emptyset$. Let $b=q^{\prime \prime \prime}+n \in\left(q^{\prime \prime \prime}+N\right) \cap B$ for some $n \in N$. Since $B$ is a subtractive extension of $N, q^{\prime \prime \prime} \in B$. So $a \in q^{\prime \prime \prime}+N \subseteq B$. Thus $\widetilde{A}$ is the smallest subtractive extension of $N$ containing $A$.

Theorem 2.4. Let $N$ be a $Q$-subsemimodule of an $R$-semimodule $M$. If $A, B$ are subsemimodules of $M$ containing $N$, then

1) $A$ is a subtractive extension of $N \Leftrightarrow A=\widetilde{A}$

2) $\widetilde{\widetilde{A}}=\widetilde{A}$

3) $A \subseteq B \Rightarrow \widetilde{A} \subseteq \widetilde{B}$

4) $A=B \Rightarrow \widetilde{A}=\widetilde{B}$.

Proof. Trivial.

In the above theorem, reverse implications are not true.

Example 2.5. Consider $M=\left(\mathbb{Z}_{0}^{+},+\right)$a semimodule over $R=\left(\mathbb{Z}_{0}^{+}\right.$, $+, \cdot)$.

1) Let $N=8 \mathbb{Z}_{0}^{+}$be a $Q$-subsemimodule of $M, A=4 \mathbb{Z}_{0}^{+}, B=$ $\{0,6,8,10, \ldots\}$. Then $A, B$ are subsemimodules of $M$ with $N \subseteq$ $A, B$ and $\widetilde{A}=4 \mathbb{Z}_{0}^{+} \subseteq 2 \mathbb{Z}_{0}^{+}=\widetilde{B}$ but $A$ is not a subset of $B$. 
2) Let $N=6 \mathbb{Z}_{0}^{+}$be a $Q$-subsemimodule of $M, A=2 \mathbb{Z}_{0}^{+}, B=$ $\{0,6,8,10, \ldots\}$. Then $A, B$ are subsemimodules of $M$ with $A \neq B$ and $N \subseteq A, B$. Here $\widetilde{A}=2 \mathbb{Z}_{0}^{+}=\widetilde{B}$.

Chaudhari and Bonde [3, Lemma 3.4], proved that if $A, N$ are subsemimodules of an $R$-semimodule $M$ with $N$ a $Q$-subsemimodule and $N \subseteq A$, then $N$ is a $Q \cap A$-subsemimodule of $A$. However, we prove the following lemma which gives a characterization of a subtractive extension of a $Q$-subsemimodule $N$ of an $R$-semimodule $M$ and will be used in the subsequent theorem.

Lemma 2.6. Let $N$ be a $Q$-subsemimodule of an $R$-semimodule $M$ and $A$ be a subsemimodule of $M$ with $N \subseteq A$. Then $A$ is a subtractive extension of $N$ if and only if $N$ is a $Q \cap A$-subsemimodule of $A$.

Proof. Let $q_{0}+N$ be the zero element of $M / N_{(Q)}$. Let $A$ be a subtractive extension of $N$ and $a \in A$. Then there exists unique $q \in Q$ such that $a \in q+N$. So $a=q+n$ for some $n \in N$. Since $A$ is a subtractive extension of $N, q \in A$. Hence $q \in Q \cap A$. If $\left(q_{1}+N\right) \cap\left(q_{2}+N\right) \neq \emptyset$ for some $q_{1}, q_{2} \in Q \cap A$, then $q_{1}=q_{2}$ because $N$ is a $Q$-subsemimodule of $M$. Thus $N$ is a $Q \cap A$-subsemimodule of $A$. Conversely suppose that $N$ is a $Q \cap A$-subsemimodule of $A$. Let $x \in N, x+y \in A$ for some $y \in M$. Since $N$ is a $Q \cap A$-subsemimodule of $A$ and $N$ a $Q$-subsemimodule of $M$ there exist unique $q_{1} \in Q \cap A, q_{2} \in Q$ such that $x+y \in q_{1}+N$ and $y \in q_{2}+N$. But then $x+y \in\left(q_{0}+N\right) \oplus\left(q_{2}+N\right)=q_{2}+N$, since $x \in N=q_{0}+N$. So $\left(q_{1}+N\right) \cap\left(q_{2}+N\right) \neq \emptyset$. Hence $q_{2}=q_{1} \in A$. Now $y \in q_{2}+N \subseteq A$.

Theorem 2.7. Let $N$ be a $Q$-subsemimodule of an $R$-semimodule $M$ and $A$ a subsemimodule of $M$. Then the following statements are equivalent:

1) $A$ is a subtractive extension of $N$

2) $A / N_{(Q \cap A)}$ is a subsemimodule of an $R$-semimodule $M / N_{(Q)}$

3) $A / N_{(Q \cap A)} \subseteq M / N_{(Q)}$

Proof. (1) $\Rightarrow(2)$ Let $A$ be a subtractive extension of $N$. By Lemma 2.6, $N$ is a $Q \cap A$-subsemimodule of $A$. Let $q_{1}+N, q_{2}+N \in A / N_{(Q \cap A)}$. Therefore $q_{1}, q_{2} \in Q \cap A \subseteq Q$. Hence there exists a unique $q_{3} \in Q$ such that $\left(q_{1}+N\right) \oplus\left(q_{2}+N\right)=q_{3}+N$ where $q_{1}+q_{2}+N \subseteq q_{3}+N$. Now $q_{1}+q_{2}=q_{3}+n$ for some $n \in N$. But $q_{3}+n=q_{1}+q_{2} \in A$. Since $A$ is a subtractive extension of $N, q_{3} \in A$. Now $q_{3}+N \in A / N_{(Q \cap A)}$. Similarly, if $r \in R, q+N \in A / N_{(Q \cap A)}$, then $r \odot(q+N) \in A / N_{(Q \cap A)}$. Hence $A / N_{(Q \cap A)}$ is a subsemimodule of $M / N_{(Q)}$. 
(2) $\Rightarrow(3)$ Trivial.

$(3) \Rightarrow(1)$ Let $x \in N, x+y \in A$ where $y \in M$. Then $x \in N=q_{0}+N$ where $q_{0}+N$ is the zero element of $M / N_{(Q)}$ and hence zero element of $A / N_{(Q \cap A)}$. Now there exist unique $q_{1} \in Q \cap A, q_{2} \in Q$ such that $x+y \in$ $q_{1}+N$ and $y \in q_{2}+N$. But then $x+y \in\left(q_{0}+N\right) \oplus\left(q_{2}+N\right)=q_{2}+N$. So $q_{1}, q_{2} \in Q$ are such that $\left(q_{1}+N\right) \cap\left(q_{2}+N\right) \neq \emptyset$. Hence $q_{1}=q_{2} \in A$. So $y \in q_{2}+N \subseteq A$.

If $N$ is a $Q$-subsemimodule of an $R$-semimodule $M$, then following theorem gives a relationship between subsemimodules of the quotient semimodule $M / N_{(Q)}$ and subsemimodules of $M$ which are subtractive extensions of $N$.

Theorem 2.8. Let $N$ be a $Q$-subsemimodule of an $R$-semimodule $M$. Then a subset $L$ of $M / N_{(Q)}$ is a subsemimodule of $M / N_{(Q)}$ if and only if there exists a subsemimodule $A$ of $M$ such that $A$ is a subtractive extension of $N$ and $A / N_{(Q \cap A)}=L$.

Proof. Let $L$ be a subsemimodule of an $R$-semimodule $M / N_{(Q)}$. Denote $A=\{x \in M$ : there exists a unique $q \in Q$ such that $x+$ $N \subseteq q+N \in L\}$, clearly $N \subseteq A$. Let $x, y \in A, r \in R$. Then there exist unique $q_{1}, q_{2} \in Q$ such that $x+N \subseteq q_{1}+N \in L, y+N \subseteq q_{2}+N \in L$. Again there exist unique $q_{3}, q_{4} \in Q$ such that $\left(q_{1}+N\right) \oplus\left(q_{2}+N\right)=$ $q_{3}+N \in L$ and $r \odot\left(q_{1}+I\right)=q_{4}+N \in L$ where $q_{1}+q_{2}+N \subseteq q_{3}+N$ and $r q_{1}+N \subseteq q_{4}+N$. By Lemma 1.2, $x+y \in x+y+N \subseteq q_{1}+q_{2}+N \subseteq q_{3}+N$ and $r x \in r x+N \subseteq r q_{1}+N \subseteq q_{4}+N$. So $x+y, r x \in A$. Hence $A$ is a subsemimodule of $M$ with $N \subseteq A$. Now let $x \in N, x+y \in A, y \in M$. So there exists a unique $q \in Q$ such that $x+y \in q+N \in L$. Since $N$ is a $Q$ subsemimodule of $M$, there exists a unique $q^{\prime} \in Q$ such that $y \in q^{\prime}+N$. Since $x \in N, x+y \in q^{\prime}+N$. So $(q+N) \cap\left(q^{\prime}+N\right) \neq \emptyset \Rightarrow q=q^{\prime}$. Now $y \in q^{\prime}+N=q+N \in L$. Thus $y \in A$. Hence $A$ is a subtractive extension of $N$. Clearly $A / N_{(Q \cap A)} \subseteq L$. Now if $q+N \in L$, then $q \in A$. So $L \subseteq A / N_{(Q \cap A)}$. Thus $A / N_{(Q \cap A)}=L$. Conversely, suppose that $A$ is a subtractive extension of $N$ and $A / N_{(Q \cap A)}=L$. Then by Theorem 2.7, $L$ is a subsemimodule of $M / N_{(Q)}$.

Corollary 2.9. Let $N$ be a $Q$-subsemimodule of an $R$-semimodule $M$ and let $T, L$ be subtractive extensions of $N$. Then $T / N_{Q}=L / N_{Q}$ if and only if $T=L$.

Proof. Let $T / N_{Q}=L / N_{Q}$ and $a \in T$. Then $a \in q_{1}+N$ for some unique $q_{1} \in Q$. So $a=q_{1}+c$ for some unique $c \in N$. Since $T$ is a subtractive extension of $N, q_{1} \in T$. Thus $q_{1}+N \subseteq T / N_{Q}=L / N_{Q}$. 
It follows that $q_{1} \in L$. Now $a=q_{1}+c \in L$. Hence $T \subseteq L$. Similarly $L \subseteq T$. Converse is trivial.

The following theorem gives a relationship between prime subsemimodules of $M$ which are subtractive extensions of a $Q$-subsemimodule $N$ of $M$ and prime subsemimodules of the quotient semimodule $M / N_{(Q)}$.

Theorem 2.10. Let $N$ be a $Q$-subsemimodule of an $R$-semimodule $M$ and $P$ be a subtractive extension of $N$. Then $P$ is a prime subsemimodule of $M$ if and only if $P / N_{(Q \cap P)}$ is a prime subsemimodule of $M / N_{(Q)}$.

Proof. Let $P$ be a prime subsemimodule of $M$. Suppose that $r \in$ $R, q_{1}+N \in M / N_{(Q)}$ are such that $r \odot\left(q_{1}+N\right)=q_{2}+N \in P / N_{(Q \cap P)}$ where $q_{2} \in Q \cap P$ is unique such that $r q_{1}+N \subseteq q_{2}+N$. Therefore $r q_{1}=q_{2}+n \in P$ for some $n \in N \subseteq P$. As $P$ is a prime subsemimodule, either $r M \subseteq P$ or $q_{1} \in P$. If $q_{1} \in P$, then $q_{1} \in Q \cap P$ and hence $q_{1}+N \in P / N_{(Q \cap P)}$. Suppose that $r M \subseteq P$. For $q+N \in M / N_{(Q)}$, let $r \odot(q+N)=q_{3}+N$ where $q_{3}$ is a unique element of $Q$ such that $r q+N \subseteq q_{3}+N$. Therefore $r q=q_{3}+n^{\prime} \in P$ for some $n^{\prime} \in N$, since $P$ is a subtractive extension of $N, q_{3} \in P$. So $q_{3} \in Q \cap P$. Now $r \odot(q+N)=q_{3}+N \in P / N_{(Q \cap P)}$ and hence $r \odot M / N_{(Q)} \subseteq P / N_{(Q \cap P)}$. So $P / N_{(Q \cap P)}$ is a prime subsemimodule of $M / N_{(Q)}$. Conversely suppose that $P / N_{(Q \cap P)}$ is a prime subsemimodule of $M / N_{(Q)}$. Let $r m \in P$ where $r \in R, m \in M$. Now there exists a unique $q_{1} \in Q$ such that $m \in q_{1}+N$ and $r m \in r \odot\left(q_{1}+N\right)=q_{2}+N$ where $q_{2}$ is a unique element of $Q$ such that $r q_{1}+N \subseteq q_{2}+N$. Now $r m \in P, r m \in q_{2}+N$ implies $q_{2} \in P$, as $P$ is a subtractive extension of $N$. Hence $r \odot\left(q_{1}+N\right)=q_{2}+N \in P / N_{(Q \cap P)}$. As $P / N_{(Q \cap P)}$ is a prime subsemimodule, either $r \odot M / N_{(Q)} \subseteq P / N_{(Q \cap P)}$ or $q_{1}+N \in P / N_{(Q \cap P)}$. If $q_{1}+N \in P / N_{(Q \cap P)}$, then $q_{1} \in P$. Hence $m \in$ $q_{1}+N \subseteq P$. So assume that $r \odot M / N_{(Q)} \subseteq P / N_{(Q \cap P)}$. For $x \in M$, there exists a unique $q_{3} \in Q$ such that $x \in q_{3}+N$ and $r x \in r \odot\left(q_{3}+N\right)=q_{4}+N$ where $q_{4}$ is a unique element of $Q$ such that $r q_{3}+N \subseteq q_{4}+N$. Now $q_{4}+N=r \odot\left(q_{3}+N\right) \in P / N_{(Q \cap P)} \Rightarrow q_{4} \in P$. Now $r x \in q_{4}+N \subseteq P$. So $r M \subseteq P$. Hence $P$ is a prime subsemimodule of $M$.

Adopting the proof of Theorem 2.10, we have the following theorems.

Theorem 2.11. Let $N$ be a $Q$-subsemimodule of an $R$-semimodule $M$ and $P$ a subtractive extension of $N$. Then $P$ is a primary subsemimodule of $M$ if and only if $P / N_{(Q \cap P)}$ is a primary subsemimodule of $M / N_{(Q)}$.

Theorem 2.12. Let $N$ be a $Q$-subsemimodule of an $R$-semimodule $M$ and $P$ be a subtractive extension of $N$. Then 
1) If $P$ is a weakly prime (weakly primary) subsemimodule of $M$, then $P / N_{(Q \cap P)}$ is a weakly prime (weakly primary) subsemimodule of $M / N_{(Q)}$.

2) If $N$ and $P / N_{(Q \cap P)}$ are weakly prime (weakly primary) subsemimodules of $M$ and $M / N_{(Q)}$ respectively, then $P$ is a weakly prime (weakly primary) subsemimodule of $M$.

\section{References}

[1] P. J. Allen, A fundamental theorem of homomorphism for semirings, Proc. Amer. Math. Soc. 21 (1969), 412-416.

[2] R. E. Atani, S. E. Atani, On subsemimodules of semimodules, Buletinul Acad. Sci. Republ. Moldova, ser. Math., Number 2 (63) (2010), 20-30.

[3] J. N. Chaudhari and D. R. Bonde, On Partitioning and Subtractive Subsemimodules of Semimodules over Semirings, Kyungpook Math. J. 50 (2010), 329336.

[4] J. N. Chaudhari and D. R. Bonde, weakly prime subsemimodules of semimodules over semirings, International J. Algebra, 5 (2011), no. 1-4, 167-174.

[5] J. N. Chaudhari and D. R. Bonde, On direct sum of partitioning subsemimodules of semimodules over semirings, Journal of Advanced Research in Pure Mathematics, 4 (2012), Issue. 1, 81-88.

[6] J. S. Golan, Semiring and their applications, Kluwer Academic publisher Dordrecht, 1999.

[7] V. G. and J. N. Chaudhari, Characterization of weakly prime subtractive ideals in semirings, Bull. Inst. Math. Acad. Sinica (New Series) 3 (2008), 347-352.

[8] G. Yesilot, On prime and maximal $k$-subsemimodules of semimodules, Hacettepe Journal of Mathematics and Statistics, 39 (3) (2010), 305-312.

[9] G. Yesilot, K. H. Oral and U. Tekir, On prime subsemimodules of semimodules, International Journal of Algebra, 4 (2010), no. 1, 53-60.

$*$

Department of Mathematics

M. J. College,

Jalgaon-425 002, India

E-mail: jnchaudhari@rediffmail.com

$* *$

Department of Mathematics

ACS College,

Dharangaon-425 105, India

E-mail: drbonde@rediffmail.com 